\title{
Enhanced Crystal Stabilities of $\varepsilon-C L-20$ via Core-Shell Structured Energetic Composites
}

\author{
Honglei Zhang ${ }^{1}$, Qingjie Jiao ${ }^{1}$, Wanjun Zhao ${ }^{1}$, Xueyong Guo ${ }^{1, *}$, Dayong $\mathrm{Li}^{2}$ and Xiaole Sun ${ }^{1}$ \\ 1 State Key Laboratory of Explosion Science and Technology, Beijing Institute of Technology, Beijing 100081, \\ China; yxzhanghonglei@163.com (H.Z.); jqj@bit.edu.cn (Q.J.); wanjunzhaowj@gmail.com (W.Z.); \\ sunxiaole060420122@126.com (X.S.) \\ 2 China North Chemical Research Academy Group Co., Ltd., Beijing 100089, China; wolanwoting@163.com \\ * Correspondence: nust@bit.edu.cn; Tel.: +86-010-6891-4863
}

Received: 5 March 2020; Accepted: 10 April 2020; Published: 12 April 2020

check for updates

Featured Application: We aimed to obtain a dense $\varepsilon$-CL-20/polydopamine core-shell structure by chemical bionic technology that can effectively inhibit the solid-solid phase transition existing for CL-20 crystals and greatly enhance the thermal safety. Obtained well-coated CL-20 particles could be employed in high-energy insensitive polymer bonded explosives (PBX), thus promoting the development of weapons and equipment.

\begin{abstract}
CL-20) is a widely used high-energy explosive for the application of energetic materials. However, the phase transformation from $\varepsilon$-CL-20 to $\gamma$-CL-20 restrains its further application in polymer bonded explosives (PBXs) and propellants. To inhibit the phase transition of CL-20, dopamine was first used in an efficient and facile method of in situ polymerization to passivate CL-20 crystals. The core-shell microcapsule particles were obtained, and the morphological characterization demonstrates the formation of a dense core-shell structure. The differential scanning calorimetry (DSC) and in situ X-ray diffraction (XRD) test results show that the compact and dense coating delays the $\varepsilon$-CL-20 crystal transformation temperature by about $30^{\circ} \mathrm{C}$, which enhances thermal stability. In addition, with the coating via polymers, the friction sensitivity of $\varepsilon$-CL-20 crystals decreases significantly. The findings indicate a successful application of dopamine chemistry in high-energy explosives, which provides an attractive method to modify the properties of CL-20 crystals.
\end{abstract}

Keywords: energetic crystal; phase transition; CL-20; dopamine; core-shell structure

\section{Introduction}

2,4,6,8,10,12-hexanitro-2,4,6,8,10,12-hexaazaisowurtzitane (CL-20) is one of the most promising explosives and propellant formulations that was synthesized first by the Naval Weapons Center, China Lake, in 1987 with higher detonation performance than conventional nitramine explosives including cyclotrimethylenetrinitramine (RDX) and cyclotetramethylenetetranitramine (HMX) $[1,2]$. LX-19 ( $\varepsilon$-CL-20 95.2\%, wax 4.8\%) is more powerful relative to the presently widely used LX-14 formulation consisting of 95.5\% HMX and 4.5\% wax, even though LX-19 is slightly more sensitive compared with LX-14 [3]. Five polymorphic phases exist for CL-20: $\varepsilon, \beta, \alpha$, and $\gamma$ phases at ambient temperature, and $\zeta$ phase at high pressure [4]. The energy density of $\varepsilon$-CL-20 is the highest with the lowest sensitivity [5], and under certain conditions, such as a certain thermal stimulus or mechanical shock, $\varepsilon$-CL-20 transfers into other phases [6]. The solid-solid phase transition of CL-20 is the transition of $\varepsilon$ into $\gamma$ phase under thermal stimulation. The rapid phase transition described above can lead to an expansion in volume, causing stress cracking. The resulting defects may induce hot spots and the 
sensitization of explosives to deflagration. The internal defect structure of explosive crystals adversely influences the mechanical and thermal sensitivity of polymer bonded explosives (PBX) $[7,8]$. Therefore, the phase transition of CL-20 must be investigated.

In recent years, the research on crystal phase transition of $\varepsilon$-CL-20 has mainly focused on two aspects: (1) the phase transition of $\varepsilon$-CL-20 during the recrystallization in a liquid-phase environment. Crystallization parameters, such as temperature, solvents, agitation rate, and the methods of adding solvents, can significantly affect the polymorphs and transition of $\varepsilon$-CL-20 [9-14]. (2) Additives are incorporated via conventional coating or physical mixing to investigate the phase transformation of $\varepsilon$-CL-20 crystals. The additives usually have a high dipole moment or slightly dissolve CL-20, which can easily polarize $\varepsilon$-CL-20, thus promoting its transition to $\gamma$-CL-20 $[15,16]$. Neutral polymers, such as glycidyl azide polymer (GAP), isocyanate desmodur (N100), ethylene propylene diene monomer (EPDM), and butadiene rubber (BR), inhibit the crystal change of CL-20 [6,17].

Despite the conventional coating method improving the coverage of the explosive particle surface to a certain extent, this method cannot completely remove the defects of the particle surface coating [10-12]. Thus, new materials must be found with strong adhesion for modifying energetic crystal particles. Recently, mussel coatings inspired by dopamine in situ polymerization attracted interest and are usually used to passivate large amounts of substrates such as glass, metals, polymers, and nano-carbon materials [18-25]. Dopamine chemistry was reported for crystal modification of energetic materials, such as HMX, RDX, and 1,3,5-triamino-2,4,6-trinitrobenzene (TATB) [11,26-28]. However, few studies have reported the application of dopamine chemistry technology for CL-20 solid-solid phase transition studies.

In this study, a convenient in-situ polymerization method was applied to prepare the core-shell $\varepsilon$-CL-20/polydopamine ( $\varepsilon$-CL-20/PDA) microparticles. The obtained core-shell structure significantly improves the thermal stability, phase transition stability, and safety of $\varepsilon$-CL-20 crystal. Four kinds of dopamine materials were selected and employed for coating experiments. Scanning electron microscopy (SEM) and X-ray photoelectron spectroscopy (XPS) were used to confirm the firmness and compactness of the core-shell structure. The solid-solid crystal transformation temperature of $\varepsilon$-CL-20 and passivated crystals, which has been little studied before, was measured via differential scanning calorimetry (DSC) and in situ X-ray diffraction (in situ XRD).

\section{Experimental Section}

\subsection{Chemicals}

$\varepsilon$-CL-20 was obtained from Liaoning Qingyang Special Chemical Co., Ltd. (Liaoyang, China), which was synthesized and purified using the solvent/anti-solvent method. Four dopamine series (dopamine, levodopa, norepinephrine, and 6-hydroxydopamine) were purchased from Shanghai Macklin Biochemical Co., Ltd., Shanghai, China. Their molecular structures are shown in Figure 1. 2-amino-2-hydroxymethylpropane-1,3-diol (Tris) was provided by Shanghai Aladdin Chemical Ltd., Shanghai, China.<smiles>NCCc1ccc(O)c(O)c1CC(N)C(N)Cc1ccc(O)c(O)c1</smiles>

Figure 1. Molecule structures of (a) dopamine, (b) levodopa, (c) norepinephrine, and (d) 6-hydroxydopamine. 


\subsection{Sample Preparations}

The core-shell structure $\varepsilon$-CL-20/PDA particles were prepared as follows: $50 \mathrm{mg}$ PDA was dissolved in $300 \mathrm{~mL}$ Tris- $\mathrm{HCl}(10 \mathrm{mM}, \mathrm{pH}$ 8.5) and stirred for $10 \mathrm{~min}$. We added $100 \mathrm{~g} \varepsilon$-CL-20 crystals to this solution, followed by stirring at $25^{\circ} \mathrm{C}$ for $24 \mathrm{~h}$. Twelve hours after PDA coating, the color of $\varepsilon$-CL-20/PDA became dark brown, different from pristine white $\varepsilon$-CL-20, revealing the completeness of the PDA self-polymerization. The composite particles were filtered from the suspension and washed with ethanol several times, followed by drying at $50{ }^{\circ} \mathrm{C}$ to obtain the $\varepsilon$-CL-20/PDA particles. Figure 2 illustrates the preparation method of the core-shell structure. The preparation methods of the other three core-shell structures ( $\varepsilon$-CL-20/poly-L-dopamine (PLD), $\varepsilon$-CL-20/poly-norepinephrine (PNE), and $\varepsilon$-CL-20/poly-6-hydroxydopamine (POHDA)) were similar to the above method.

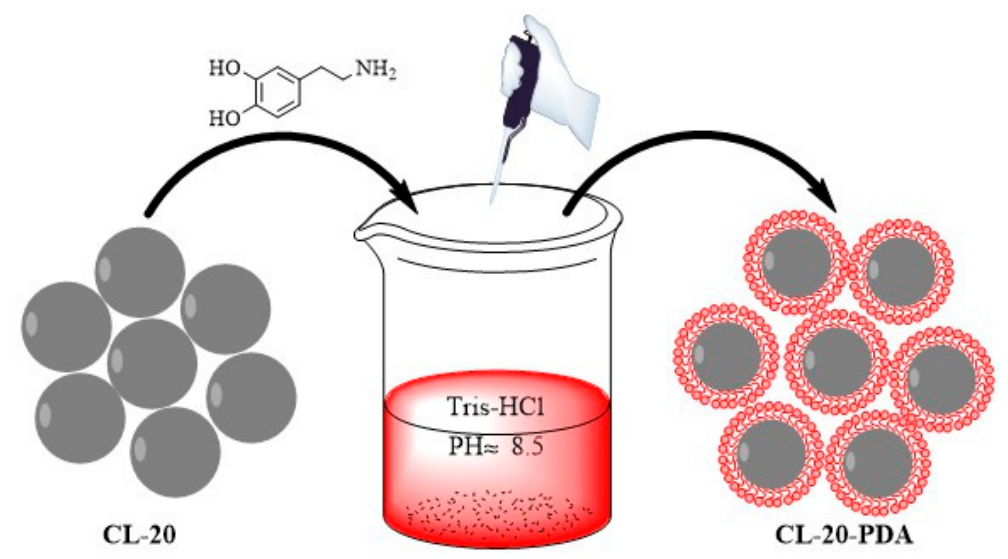

Figure 2. The schematic diagram of the preparation method of the core-shell structure.

\subsection{Characterization Method}

The microstructure and the electronic binding energy of the elements of raw $\varepsilon$-CL-20, $\varepsilon$-CL-20/PDA, $\varepsilon$-CL-20/PLD, $\varepsilon$-CL-20/PNE, and $\varepsilon$-CL-20/POHDA were characterized via SEM and XPS. DSC (STA449C, Netzsch, Selb, Germany) and in situ XRD (Bruker, Karlsruhe, Germany) were used to evaluate the crystal transformation. In a typical DSC, $\sim 5 \mathrm{mg}$ of sample was heated from ambient temperature to $450{ }^{\circ} \mathrm{C}$ with a heating rate of $5{ }^{\circ} \mathrm{C} / \mathrm{min}$ in an Ar atmosphere. In situ XRD was conducted on a Bruker D8 Advance (Bruker, Karlsruhe, Germany) diffractometer ( $\mathrm{Cu} \mathrm{K} \alpha$ radiation, $40 \mathrm{kV}$, and $40 \mathrm{~mA}$ ), scanning over the $2 \theta$ range from $5^{\circ}$ to $50^{\circ}$ at a rate of $0.01^{\circ} / 0.1 \mathrm{~s}$. To determine the crystalline phase of compositions, the obtained spectra data were refined using the Rietveld method [29]. The mechanical sensitivity was characterized via a WL-1 type impact sensitivity instrument and a WM-1 type friction sensitivity instrument [30]. The impact sensitivity was tested with a $2 \mathrm{~kg}$ drop weight, and the sample mass was $30 \mathrm{mg}$. The impact sensitivity of each test sample is expressed by the drop height of $50 \%$ explosion probability $\left(\mathrm{H}_{50}\right)$. For the impact sensitivity test, a $20 \mathrm{mg}$ sample was weighed for each test at the relative pressure of $2.54 \mathrm{MPa}$. The pendulum weight was $1.5 \mathrm{~kg}$ and the pendulum angle was $80^{\circ}$. The fraction sensitivity of each test sample is expressed by explosion probability $(\mathrm{P})$.

\section{Results and Discussion}

\subsection{Morphology and Characteristics}

The polymer content in the composites was tested by high-performance liquid chromatography (HPLC). Table 1 shows that the polymers concentrations for $\varepsilon$-CL-20/PDA, $\varepsilon$-CL-20/PLD, $\varepsilon$-CL-20/PNE, and $\varepsilon$-CL-20/POHDA composites were $1.6,0.7,1.3$, and $1.4 \mathrm{wt} . \%$, respectively. The morphologies of raw $\varepsilon$-CL-20 and four core-shell structures ( $\varepsilon$-CL-20/PDA, $\varepsilon$-CL-20/PLD, $\varepsilon$-CL-20/PNE, and $\varepsilon$-CL-20/POHDA) are shown in Figure 3. For the raw $\varepsilon$-CL-20 particles prepared via a recrystallization process, the crystal shape was a more regular polyhedron (Figure $3 \mathrm{f}$ ). The shape of the passivated $\varepsilon$-CL-20 crystal particles 
remains polyhedral. To compare the surfaces of raw $\varepsilon$-CL-20 and core-shell structures, SEM images with high magnification are shown in Figure 3a-e. The white CL-20 crystals gradually turned brown-black with progressing polymerization. After $24 \mathrm{~h}$ of reaction, the color of the crystal particles did not change, indicating the polymerization finished. As shown in Figure $3 a-d$, four polymers in a dense protective film formed on the surface of the $\varepsilon$-CL-20 particles. In comparison with the protuberance and cracks appearing on the surface of $\varepsilon$-CL-20 crystals (Figure 3e) caused by the electron beams during the SEM test, similar phenomena were significantly improved in the well-coated samples (Figure 3a-d). This reveals that even though the raw explosive crystals are extremely sensitive to electron beams, dense polymer films coated on the crystals could effectively improve the resistance to electron beams [25].

Table 1. Composition of core-shell microcapsules.

\begin{tabular}{ccc}
\hline Sample & $\varepsilon$-CL-20 Content (wt \%) & Content (wt \%) \\
\hline epsilon-CL-20 ( $\varepsilon$-CL-20) & 100 & 0 \\
$\varepsilon$-CL-20/polydopamine (PDA) & 98.4 & 1.6 \\
$\varepsilon$-CL-20/poly-L-dopamine (PLD) & 99.3 & 0.7 \\
$\varepsilon$-CL-20/poly-norepinephrine (PNE) & 98.7 & 1.3 \\
$\varepsilon$-CL-20/poly-6-hydroxydopamine (POHDA) & 98.6 & 1.4 \\
\hline
\end{tabular}

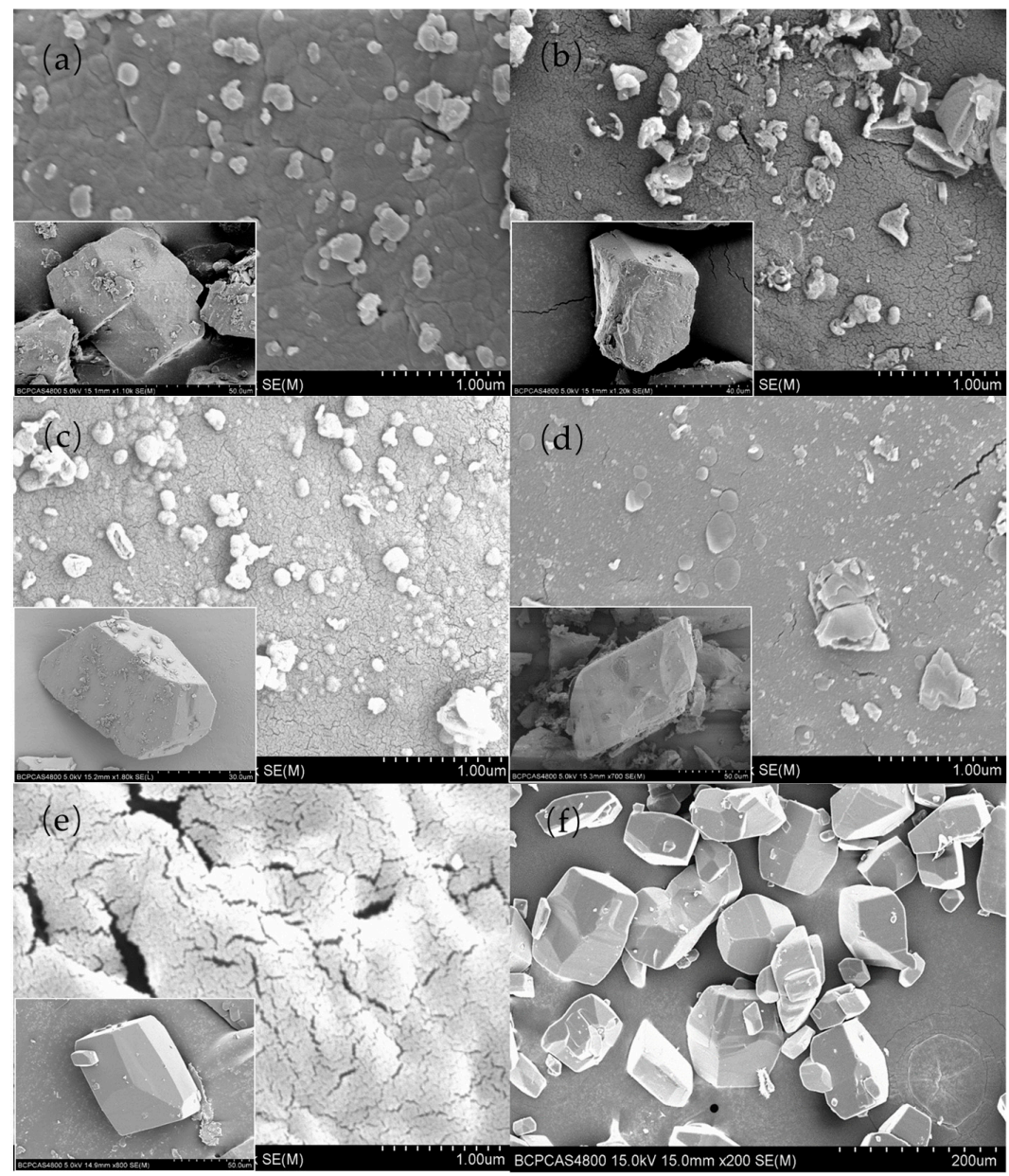

Figure 3. Scanning electron microscopy (SEM) images for (a) epsilon-CL-20 ( $\varepsilon$-CL-20)/polydopamine (PDA), (b) $\quad \varepsilon$-CL-20/poly-L-dopamine (PLD), (c) $\varepsilon$-CL-20/poly-norepinephrine (PNE), (d) $\varepsilon$-CL-20/poly-6-hydroxydopamine (POHDA), and (e,f) $\varepsilon$-CL-20. 
The change in the electronic binding energy of the elements on the surface of $\varepsilon$-CL-20 determined by the XPS test provided valuable insights into the costing effectiveness. The $\mathrm{C} 1 \mathrm{~s}, \mathrm{O} 1 \mathrm{~s}$, and $\mathrm{N} 1 \mathrm{~s}$ spectra for $\varepsilon$-CL-20, PDA, and $\varepsilon$-CL-20/PDA samples are shown in Figure 4. The spectrum data of the other three groups ( $\varepsilon$-CL-20/PLD, $\varepsilon$-CL-20/PNE, and $\varepsilon$-CL-20/POHDA samples) are shown in Figures 5-7. The percent contribution of the C1s, O1s, and N1s atom to $\varepsilon$-CL-20, PDA, and $\varepsilon$-CL-20/PDA samples were integrated and are presented in Table 2 . The coating effect of explosive particles can be accessed via analyzing the N/C ratio on the particle surface [25]. The N/C ratio on the surfaces of PDA, PLD, PNE, and POHDA are $0.11,0.13,0.12$, and 0.12 , respectively. The N/C atomic ratio of CL-20 (1.06) is relatively higher, which is due to the existence of various $-\mathrm{NO}_{2}$ and $-\mathrm{NH}_{2}$ groups. After being coated with PDA, PLD, PNE, or POHDA, the N/C ratio decreased from 1.06 to $0.51,0.70,0.63$, and 0.55, respectively, which indicated successful surface coating.
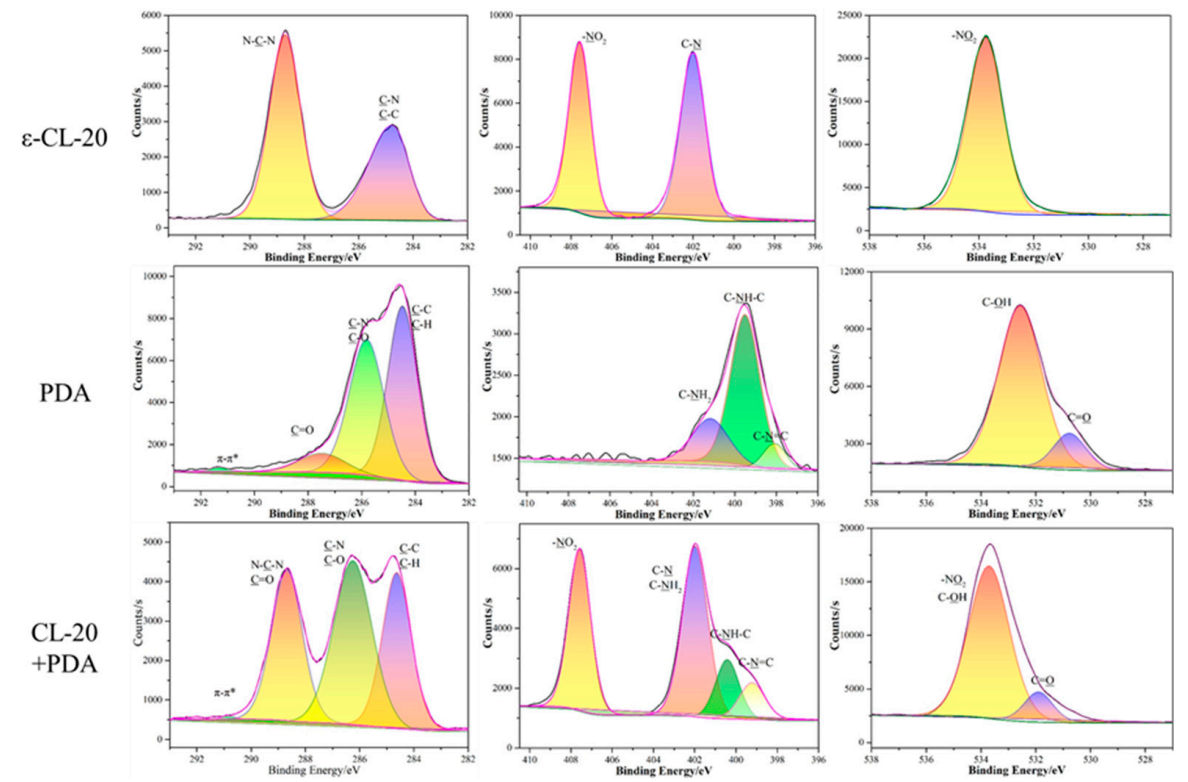

Figure 4. X-ray photoelectron spectroscopy

(XPS) spectra for $\varepsilon$-CL-20, PDA, and $\varepsilon$-CL-20/PDA composites.
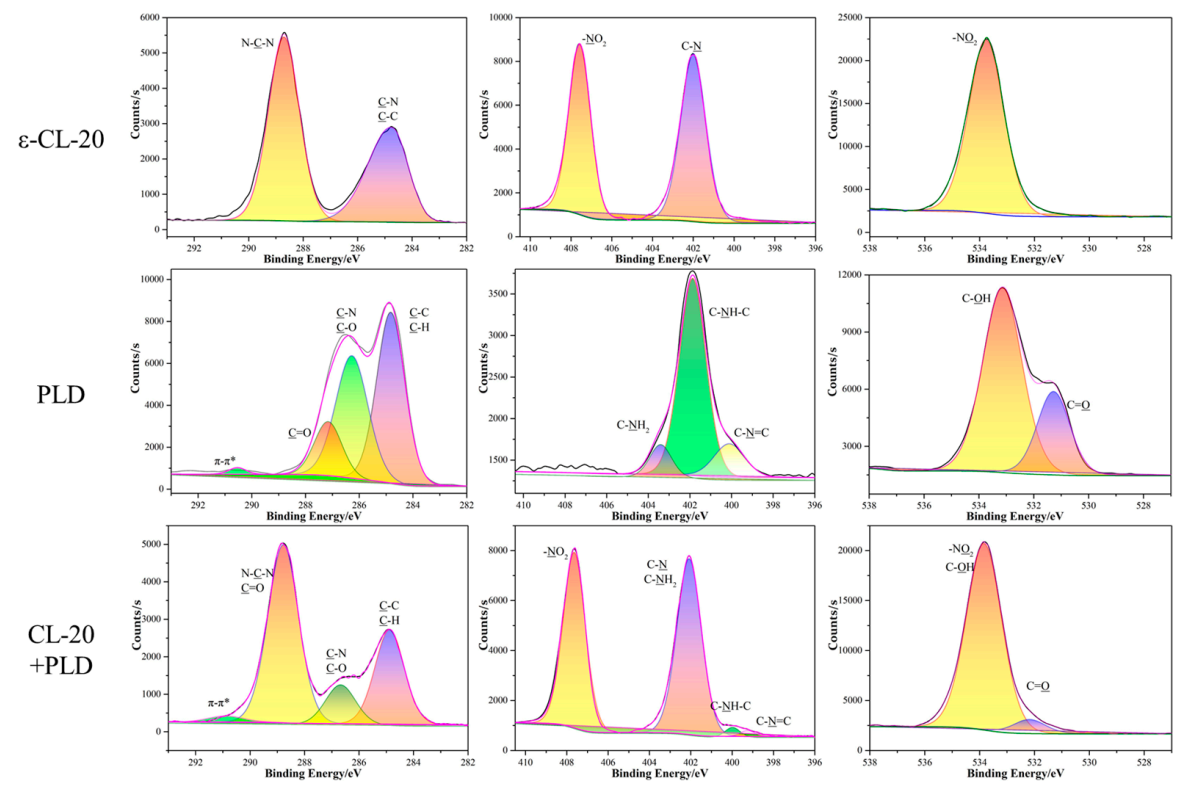

Figure 5. XPS spectra for $\varepsilon$-CL-20, PLD, and $\varepsilon$-CL-20/PLD core-shell composites. 

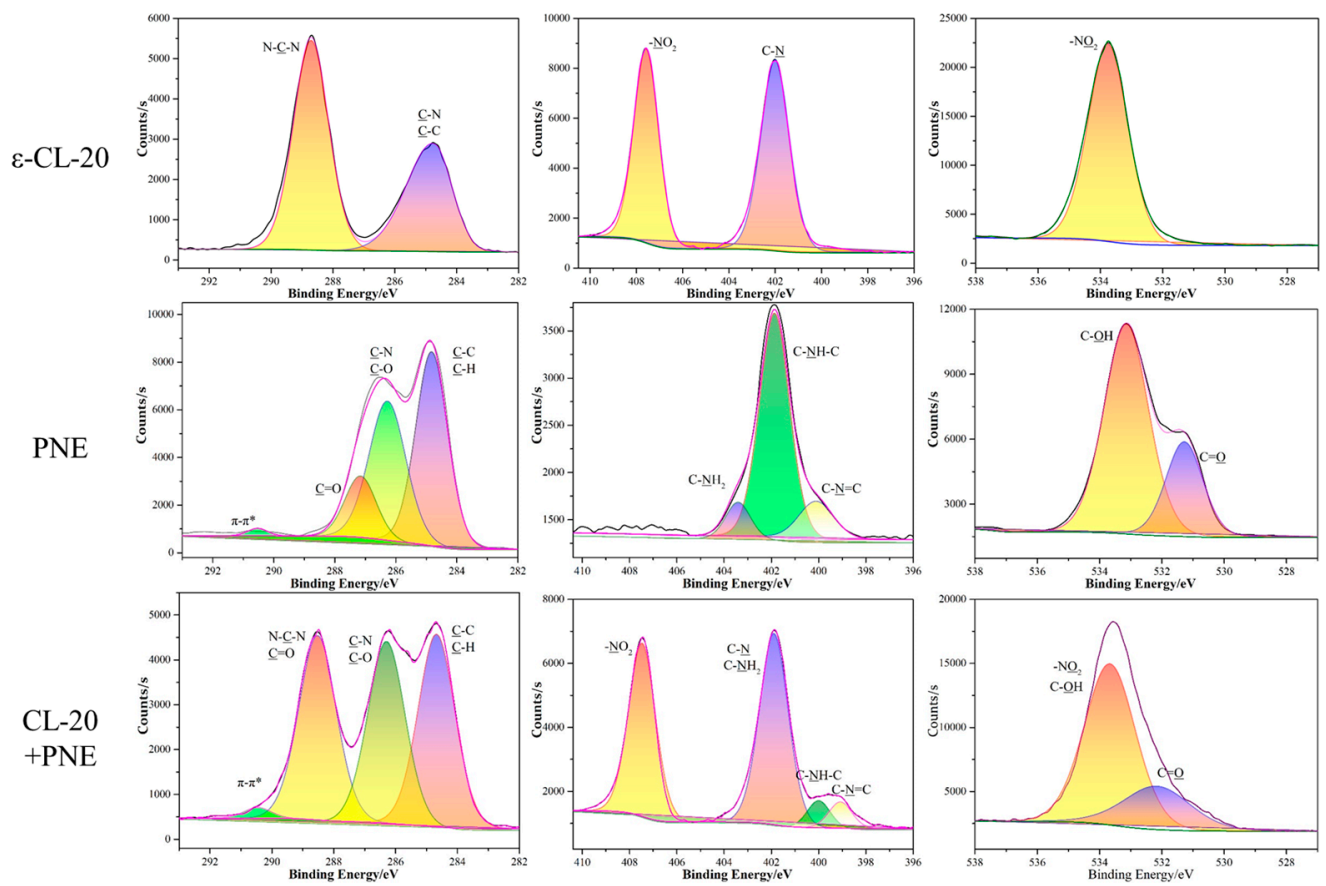

Figure 6. XPS spectra for $\varepsilon$-CL-20, PNE, and $\varepsilon$-CL-20/PNE core-shell composites.
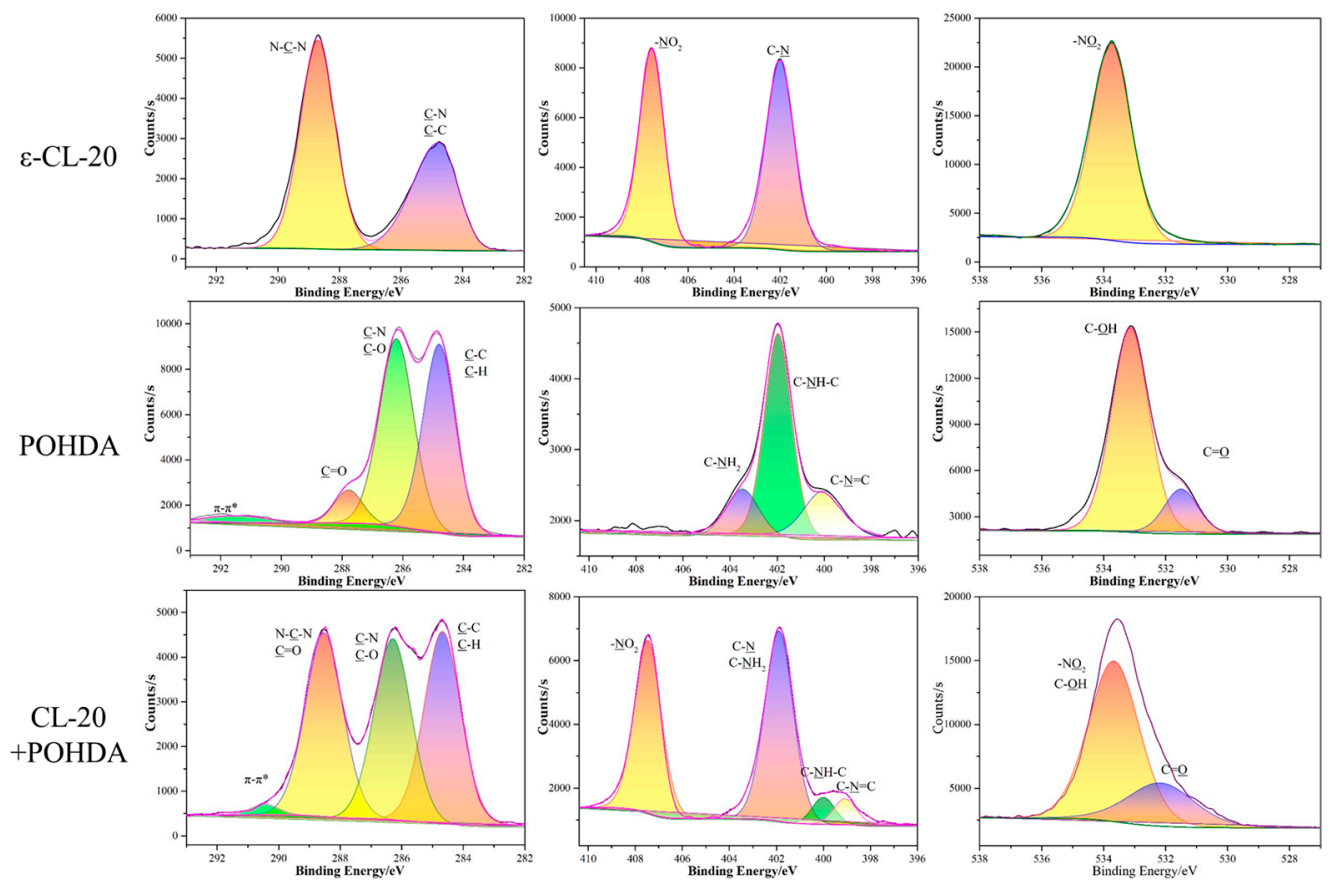

Figure 7. XPS spectra for $\varepsilon$-CL-20, POHDA, and $\varepsilon$-CL-20/POHDA core-shell composites.

From the spectrum of PDA in Figure 4, four fitted C1s peaks centered at 284.5, 285.8, 287.9, and $291.2 \mathrm{eV}$ are attributed to $\mathrm{C}-\mathrm{H}$ or $\mathrm{C}-\mathrm{C}, \mathrm{C}-\mathrm{O}$, or $\mathrm{C}-\mathrm{N}, \mathrm{C}=\mathrm{O}$, and $\pi-\pi^{*}$ shake up. Three peak spectra of N1s appeared, corresponding to $\mathrm{CN}=\mathrm{C}, \mathrm{C}-\mathrm{NH}-\mathrm{C}$, and $\mathrm{C}-\mathrm{NH}_{2}$ bonds, respectively, and their binding energies were $398.2,399.5$, and $401.3 \mathrm{eV}$, respectively. The O1s spectrum was fitted to two peaks at 530.8 and $532.7 \mathrm{eV}$, which were $\mathrm{C}=\mathrm{O}$ and $\mathrm{C}-\mathrm{OH}$, respectively. The characteristic peak of $\mathrm{C}=\mathrm{O}$ appeared in both the $\mathrm{C} 1 \mathrm{~s}$ spectrum and the $\mathrm{O} 1 \mathrm{~s}$ spectrum, indicating that during the dopamine polymerization process, the catechol group of dopamine was oxidized to a quinone group. The emerged peaks 
at 398.2 and $530.8 \mathrm{eV}$ from N1s and O1s spectra could be attributed to $\mathrm{C}-\mathrm{N}=\mathrm{C}$ and $\mathrm{C}=\mathrm{O}$ for PDA, respectively [31].

Table 2. Surface element composition of samples as evaluated by X-ray photoelectron spectroscopy (XPS).

\begin{tabular}{ccccc}
\hline Sample & C1s (\%) & N1s (\%) & O1s (\%) & N/C \\
\hline$\varepsilon$-CL-20 & 32.76 & 34.68 & 32.56 & 1.06 \\
PDA & 72.38 & 8.21 & 19.41 & 0.11 \\
PLD & 63.29 & 8.45 & 28.26 & 0.13 \\
PNE & 67.24 & 8.2 & 24.56 & 0.12 \\
POHDA & 67.72 & 8.08 & 24.2 & 0.12 \\
$\mathcal{\varepsilon}$-CL-20/PDA & 46.33 & 23.83 & 29.84 & 0.51 \\
$\mathcal{\varepsilon}-$ CL-20/PLD & 39.8 & 27.78 & 32.42 & 0.70 \\
$\varepsilon-C L-20 / P N E$ & 41.58 & 26.29 & 32.13 & 0.63 \\
$\varepsilon-C L-20 / P O H D A$ & 44.89 & 24.66 & 30.45 & 0.55 \\
\hline
\end{tabular}

\subsection{Thermal Properties of $\varepsilon$-CL-20 Composite Structures}

The thermal stability of energetic materials is an important aspect of the material properties. DSC was employed to explore the thermal behavior of $\varepsilon$-CL-20 crystals and the coated samples (Figure 8). As shown in the DSC curve of $\varepsilon$-CL-20, the endothermic and exothermic peaks appeared at 156.3 and $328.5^{\circ} \mathrm{C}$, respectively, which were attributed to the transition from $\varepsilon$ into $\gamma$ phase and the thermal decomposition of CL-20. The temperature of the thermal decomposition modified by PDA is basically the same as that of the original explosive crystal [32].

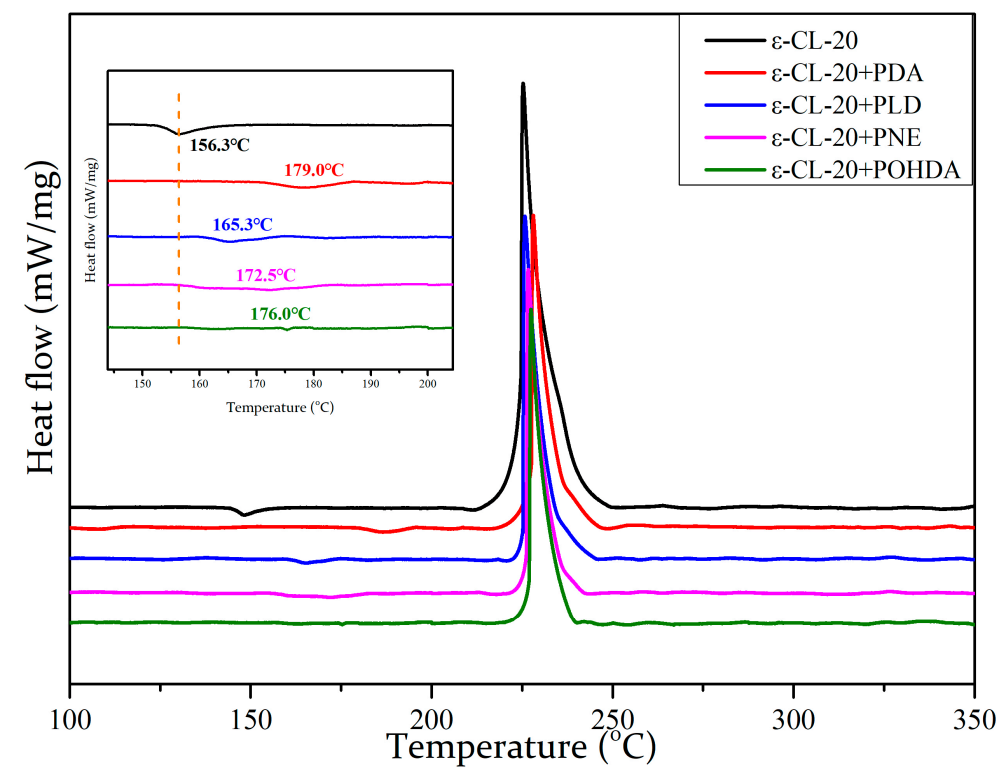

Figure 8. Differential scanning calorimetry (DSC) curves for epsilon-CL-20 ( $\varepsilon$-CL-20) and different composites.

According to the analysis in Table 3, the phase transition temperature of $\varepsilon$-CL-20 crystal coated by self-oxidizing polymerization increased considerably. In detail, the peak temperatures of $\varepsilon$-CL-20/PDA, $\varepsilon$-CL-20/PLD, $\varepsilon$-CL-20/PNE, and $\varepsilon$-CL-20/POHDA samples increased from 156.3 to 179.0, 165.3, 172.5, and $176.0^{\circ} \mathrm{C}$, respectively. The SEM images shown in Figure 3 indicate that the dense coating of PDA on each crystal may be the main reason that the small amount of PDA could inhibit the phase change significantly. Compared with the samples prepared via mechanical mixing, the initial crystal 
transformation temperature of $\varepsilon$-CL-20 after PDA self-oxidative polymerization coating increased by about $30^{\circ} \mathrm{C}$, which indicates a markedly improved thermal safety of $\varepsilon$-CL-20 during application [25].

Table 3. Thermal analysis data of epsilon-CL-20 ( $\varepsilon$-CL-20) and different composites.

\begin{tabular}{ccccc}
\hline \multirow{2}{*}{ Sample } & \multicolumn{4}{c}{ Endothermic Peak } \\
\cline { 2 - 5 } & $\boldsymbol{T}_{\boldsymbol{o}}\left({ }^{\circ} \mathbf{C}\right)$ & $\boldsymbol{T}_{\boldsymbol{p}}\left({ }^{\circ} \mathbf{C}\right)$ & $\boldsymbol{T}_{\boldsymbol{e}}\left({ }^{\circ} \mathbf{C}\right)$ & $\boldsymbol{\Delta H}\left(\mathbf{J g}^{-\mathbf{1}}\right)$ \\
\hline $\mathcal{E}$-CL-20 & 144.5 & 156.3 & 171.4 & -41.3 \\
$\mathcal{\varepsilon}$-CL-20/PDA & 172.3 & 179 & 187.1 & -28.1 \\
$\mathcal{E}$-CL-20/PLD & 157.6 & 165.3 & 174.7 & -35.8 \\
$\mathcal{E}-$ CL-20/PNE & 164.8 & 172.5 & 184.5 & -32.9 \\
$\varepsilon$-CL-20/POHDA & 173.4 & 176 & 185.2 & -30.4 \\
\hline
\end{tabular}

\subsection{Phase Transition Stability of $\varepsilon$-CL-20 Composite Structures}

Figure 9 shows the standard spectra of four polymorphs of CL-20 and the X-ray spectra of $\varepsilon$-CL-20 at various temperatures. In the diffraction angle $(2 \theta)$ ranging from $12^{\circ}$ to $14^{\circ}$, the two crystal forms of CL-20 are significantly different. In comparison with the peaks at $12.8^{\circ}$ and $13.8^{\circ}$ of $\varepsilon$-CL-20, the new peaks appear at $12.9^{\circ}, 13.3^{\circ}$, and $14.2^{\circ}$ for $\gamma$-CL-20 [6]. According to Section 2.2, four kinds of CL-20 samples coated with different polymers were prepared, and the phase transition behavior of CL-20 in the composite system was characterized by in situ XRD [4]. Figure 10 shows the $X$-ray patterns of $\varepsilon$-CL-20/PDA, $\varepsilon$-CL-20/PLD, $\varepsilon$-CL-20/PNE, and $\varepsilon$-CL-20/POHDA at different temperatures. The phase composition of the $\varepsilon$-CL-20 crystals was calculated via the Rietveld refinement method through the Topas Academy procedure, which is standardless X-ray diffraction [29]. The refinement fit test results are presented in Figure 11.
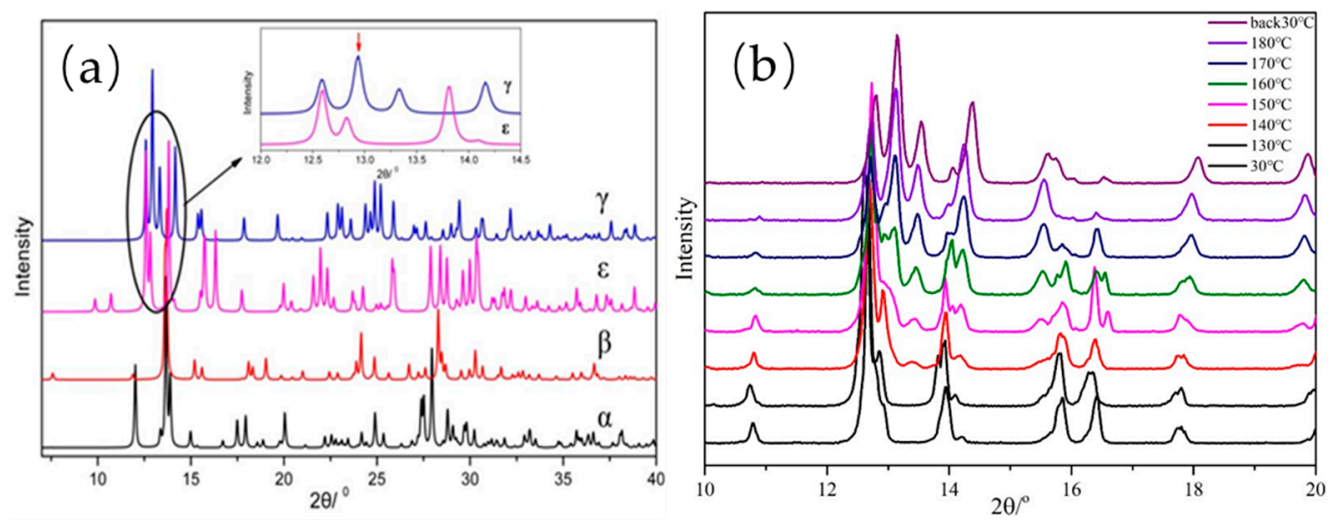

Figure 9. X-ray diffraction (XRD) patterns of (a) the conformers from four polymorphs of CL-20 and (b) $\varepsilon$-CL-20 at various temperatures.

Figures 9 and 10 show that with increasing sample temperature, the characteristic peak of $\varepsilon$-CL-20 gradually weakened, and the characteristic peak of $\gamma$-CL-20 appeared and increased, indicating more $\varepsilon$-CL-20 changed to $\gamma$-CL-20. The XRD patterns did not change when the temperature decreased from 190 to $30^{\circ} \mathrm{C}$, which demonstrated an irreversible process for the crystal form change in this situation. As shown in Figure 11, the initial crystal transformation temperatures of $\varepsilon$-CL-20, $\varepsilon$-CL-20/PDA, $\varepsilon$-CL-20/PLD, $\varepsilon$-CL-20/PNE, and $\varepsilon$-CL-20/POHDA are 145, 175, 160, 160, and 170, ${ }^{\circ} \mathrm{C}$, respectively. With the temperature increasing to $190{ }^{\circ} \mathrm{C}$, we found that almost all $\varepsilon$-CL-20 of $\varepsilon$-CL-20, $\varepsilon$-CL-20/PLD, and $\varepsilon$-CL-20/PNE converted to $\gamma$-CL-20. However, the $\gamma$-CL-20 contents of $\varepsilon$-CL-20/PDA and $\varepsilon$-CL-20/POHDA were $37.9 \%$ and $73.3 \%$, which indicated that PDA and POHDA could inhibit the CL-20 crystal transformation well. 

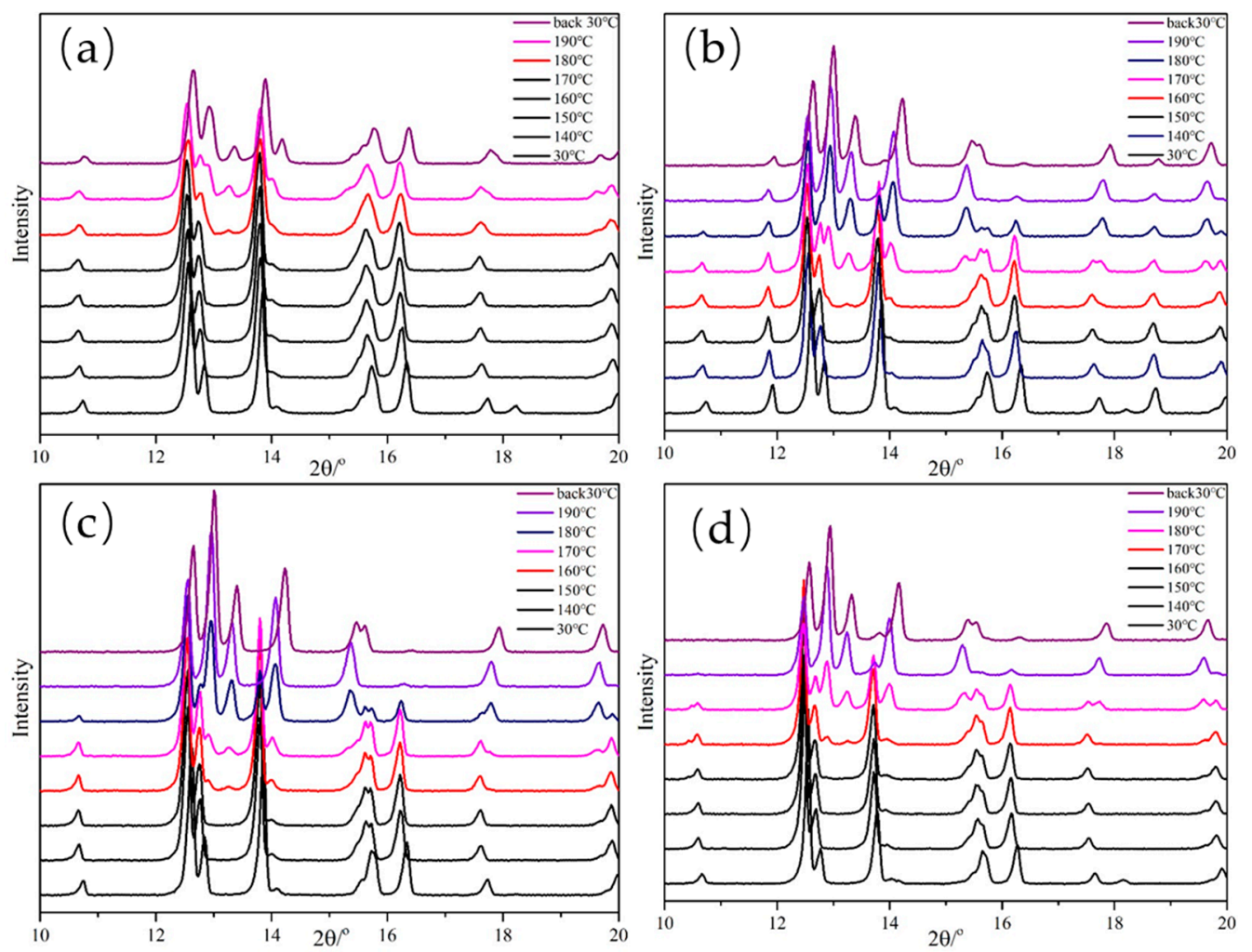

Figure 10. XRD patterns of core-shell composites at various temperatures: (a) $\varepsilon$-CL-20/PDA, (b) $\varepsilon$-CL-20/PLD, (c) $\varepsilon$-CL-20/PNE, and (d) $\varepsilon$-CL-20/POHDA.

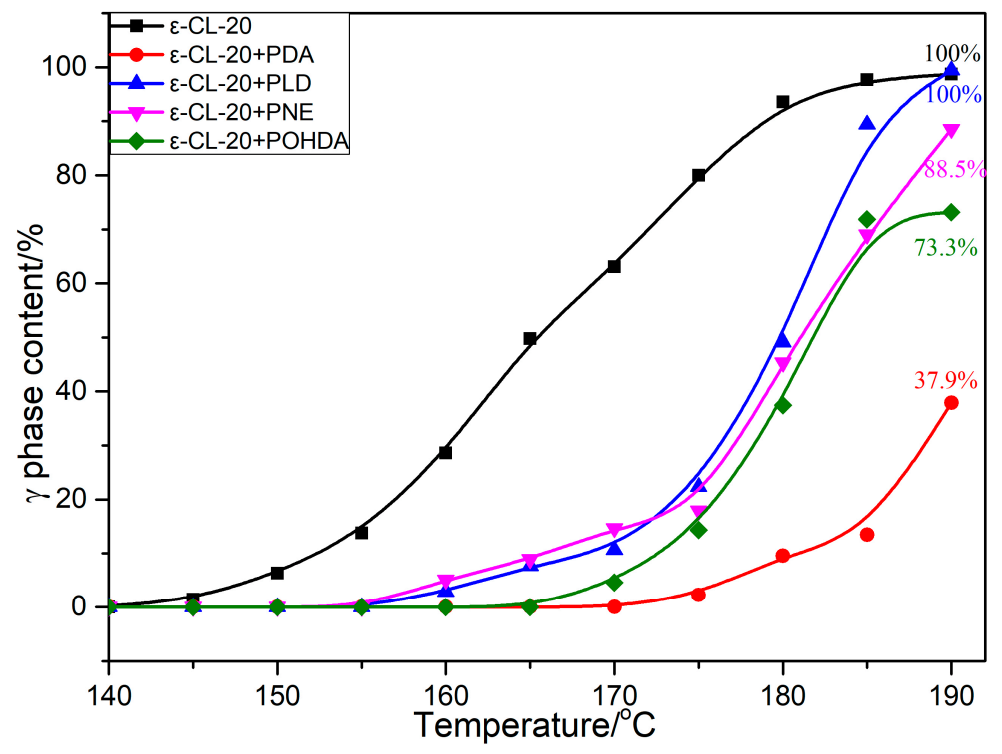

Figure 11. Phase analysis of $\varepsilon$-CL-20 and different composites.

Different from the crystallization from a solution, no polymorphic transition occurs when the medium is a solid matrix with a solvent. The activation energy for forming a new crystal phase in a solid environment is much higher than in a liquid environment, leading to a more complicated polymorphic transformation process in the solid matrix. The intense interfacial interaction between the PDA and the explosive crystal results in the high polycrystalline transition temperature. The polymorphic conversion could be reduced greatly via PDA coating, especially when the nucleation processes is the rate-limiting step [33]. 


\subsection{Mechanical Sensitivity of $\varepsilon$-CL-20 Composite Structures}

The mechanical sensitivity of explosive crystals is a key parameter for characterizing the safety of explosives. $\varepsilon$-CL-20 particles have lower mechanical sensitivity, which is beneficial for their application in weapons and equipment. According to Table 4, the friction sensitivity of $\varepsilon$-CL-20 crystals coated with PDA, PLD, PNE, and POHDA with shell-core structure decreased greatly from $96 \%$ to $48 \%, 60 \%$, $60 \%$, and $56 \%$, respectively. Among these composites, the friction sensitivity of $\varepsilon$-CL-20/PDA was the lowest. The significant decrease in friction sensitivity of $\varepsilon$-CL-20 crystals via coating may be attributed to the stronger interfacial interaction between $\varepsilon$-CL-20 crystals and polymers. However, the shell-core structure prepared by self-polymerization had no significant effect on reducing the impact sensitivity of $\varepsilon$-CL-20. The irregular shape and defects of the crystal are the main reasons for its high impact sensitivity [34]; this correspond to the results in Figure 3-the shape of $\varepsilon$-CL-20 does not change considerably after the self-polymerizing coating. We proved that a uniform and compact passivating shell was formed by PDA on the CL-20 crystals, which could help optimize the properties of energetic crystals for applications in special service environments, which is worth further research.

Table 4. Impact and friction sensitivity of $\varepsilon$-CL-20 and different composites.

\begin{tabular}{|c|c|c|}
\hline Sample & Impact Sensitivity $\left(\mathrm{H}_{50}, \mathrm{~cm}\right)$ & Friction Sensitivity $(\mathrm{P}, \%)$ \\
\hline$\varepsilon-C L-20$ & $13 \pm 1$ & 96 \\
\hline$\varepsilon-C L-20 / P D A$ & $12 \pm 1$ & 48 \\
\hline$\varepsilon-C L-20 / P L D$ & $13 \pm 1$ & 60 \\
\hline$\varepsilon-C L-20 / P N E$ & $14 \pm 1$ & 60 \\
\hline$\varepsilon-C L-20 /$ POHDA & $12 \pm 1$ & 56 \\
\hline
\end{tabular}

\section{Conclusions}

The dopamine surface chemistry was found to be a highly efficient and concise method for passivating energetic crystals, resulting in a stable core-shell CL-20/PDA structure. The structural characterization confirmed the morphology and formation mechanism of the PDA series, which is helpful for exploring the structural evolution and deposition mechanism of PDA on the $\varepsilon$-CL-20 surface. The experimental results showed that the compact and dense coating increases the $\varepsilon$-CL-20 crystal transformation temperature by about $30^{\circ} \mathrm{C}$ and significantly improves the thermal stability of $\varepsilon$-CL-20 crystals, which is important for ensuring the safety of energetic materials. With the coating via polymers, the friction sensitivity of $\varepsilon$-CL-20 crystals decreased significantly. Our findings provide new opportunities for the surface modification of high-energy explosive crystals and the preparation of energetic materials with high-level performance.

Author Contributions: Conceptualization, Q.J. and X.G.; Formal analysis, W.Z.; Supervision, X.S. and D.L.; Writing—original draft, H.Z.; Writing—review \& editing, H.Z. All authors have read and agreed to the published version of the manuscript.

Funding: This research was financially supported by the National Natural Science Foundation of China (Grant No. U1730108).

Acknowledgments: We appreciate the support from State Key Laboratory of Explosive Science.

Conflicts of Interest: The authors declare no conflict of interest.

\section{References}

1. Nielsen, A.T. Caged Polynitramine Compound. U.S. Patent No. 5,693,794, 2 December 1997.

2. Nielsen, A.T. Synthesis of Polynitropolyaza Caged Nitramines; Chemical Propulsion Information Agency: Columbia, MD, USA, 1987.

3. Simpson, R.L.; Urtiew, P.A.; Ornellas, D.L.; Moody, G.L.; Scribner, K.F.J.; Hoffman, D.M. CL-20 performance exceeds that of HMX and its sensitivity is moderate. Propellants Explos. Pyrotech. 1997, 22, 249-255. [CrossRef] 
4. Zhang, P.; Xu, J.J.; Guo, X.Y.; Jiao, Q.J.; Zhang, J.Y. Effect of addictives on polymorphic transition of epsilon-CL-20 in castable systems. J. Anal. Calorim. 2014, 117, 1001-1008. [CrossRef]

5. Ghosh, M.; Venkatesan, V.; Mandave, S.; Banerjee, S.; Sikder, N.; Sikder, A.K.; Bhattacharya, B. Probing Crystal Growth of epsilon- and alpha-CL-20 Polymorphs via Metastable Phase Transition Using Microscopy and Vibrational Spectroscopy. Cryst. Growth Des. 2014, 14, 5053-5063. [CrossRef]

6. Guo, C.; Wang, D.; Gao, B.; Wang, J.; Luo, B.; Yang, G.; Nie, F. Solid-solid phase transition study of $\varepsilon-C L-20 /$ binder composites. RSC Adv. 2016, 6, 859-865. [CrossRef]

7. Gump, J.C.; Peiris, S.M. Phase transitions and isothermal equations of state of epsilon hexanitrohexaazaisowurtzitane (CL-20). J. Appl. Phys. 2008, 104, 083509. [CrossRef]

8. Tian, Q.; Yan, G.; Sun, G.; Huang, C.; Xie, L.; Chen, B.; Huang, M.; Li, H.; Liu, Y.; Wang, J. Thermally Induced Damage in Hexanitrohexaazaisowurtzitane. Cent. Eur. J. Energ. Mater. 2013, 10, 359-369.

9. Gavezzotti, A.; Simonetta, M. Crystal-Chemistry in Organic-Solids. Chem. Rev. 1982, 82, 1-13. [CrossRef]

10. Jung, J.W.; Kim, K.J. Effect of Supersaturation on the Morphology of Coated Surface in Coating by Solution Crystallization. Ind. Eng. Chem. Res. 2011, 50, 3475-3482. [CrossRef]

11. Yang, Z.J.; Ding, L.; Wu, P.; Liu, Y.G.; Nie, F.D.; Huang, F.L. Fabrication of RDX, HMX and CL-20 based microcapsules via in situ polymerization of melamine-formaldehyde resins with reduced sensitivity. Chem. Eng. J. 2015, 268, 60-66. [CrossRef]

12. Ma, Z.G.; Gao, B.; Wu, P.; Shi, J.C.; Qiao, Z.Q.; Yang, Z.J.; Yang, G.C.; Huang, B.; Nie, F.D. Facile, continuous and large-scale production of core-shell HMX@TATB composites with superior mechanical properties by a spray-drying process. RSC Adv. 2015, 5, 21042-21049. [CrossRef]

13. Levitas, V.I.; Henson, B.F.; Smilowitz, L.B.; Asay, B.W. Solid-solid phase transformation via virtual melting significantly below the melting temperature. Phys. Rev. Lett. 2004, 92, 235702. [CrossRef] [PubMed]

14. Li, J.Q.; Ballmer, S.G.; Gillis, E.P.; Fujii, S.; Schmidt, M.J.; Palazzolo, A.M.E.; Lehmann, J.W.; Morehouse, G.F.; Burke, M.D. Synthesis of many different types of organic small molecules using one automated process. Science 2015, 347, 1221-1226. [CrossRef] [PubMed]

15. Guo, X.Y.; Zhang, P.; Xu, J.J.; Wang, Z.H.; Jiao, Q.J. Research on the Mechanism and Kinetics of Polymorphic Transitions of epsilon-CL-20 in Composite Systems. Cent. Eur. J. Energ. Mater. 2015, 12, 689-702.

16. Liu, Y.; Li, S.C.; Wang, Z.S.; Xu, J.J.; Sun, J.; Huang, H. Thermally Induced Polymorphic Transformation of Hexanitrohexaazaisowurtzitane (HNIW) Investigated by in-situ X-ray Powder Diffraction. Cent. Eur. J. Energ. Mater. 2016, 13, 1023-1037. [CrossRef]

17. Zhang, J.-Y.; Guo, X.-Y.; Jiao, Q.-J.; Zhang, P. Phase transitions of epsilon-HNIW in compound systems. AIP Adv. 2016, 6, 055016. [CrossRef]

18. Zhu, Q.; Xiao, C.; Li, S.B.; Luo, G. Bioinspired Fabrication of Insensitive HMX Particles with Polydopamine Coating. Propellants Explos. Pyrotech. 2016, 41, 1092-1097. [CrossRef]

19. Shang, K.; Song, S.Y.; Cheng, Y.P.; Guo, L.L.; Pei, Y.X.; Lv, X.M.; Aastrup, T.; Pei, Z.C. Fabrication of Carbohydrate Chips Based on Polydopamine for Real-Time Determination of Carbohydrate-Lectin Interactions by QCM Biosensor. Polymers 2018, 10, 1275. [CrossRef] [PubMed]

20. Postma, A.; Yan, Y.; Wang, Y.J.; Zelikin, A.N.; Tjipto, E.; Caruso, F. Self-Polymerization of Dopamine as a Versatile and Robust Technique to Prepare Polymer Capsules. Chem. Mater. 2009, 21, 3042-3044. [CrossRef]

21. Nie, C.X.; Yang, Y.; Cheng, C.; Ma, L.; Deng, J.; Wang, L.R.; Zhao, C.S. Bioinspired and biocompatible carbon nanotube-Ag nanohybrid coatings for robust antibacterial applications. Acta Biomater. 2017, 51, 479-494. [CrossRef]

22. Nie, C.X.; Cheng, C.; Peng, Z.H.; Ma, L.; He, C.; Xia, Y.; Zhao, C.S. Mussel-inspired coatings on Ag nanoparticle-conjugated carbon nanotubes: Bactericidal activity and mammal cell toxicity. J. Mater. Chem. B 2016, 4, 2749-2756. [CrossRef]

23. Jiang, J.H.; Zhu, L.P.; Zhu, L.J.; Zhu, B.K.; Xu, Y.Y. Surface Characteristics of a Self-Polymerized Dopamine Coating Deposited on Hydrophobic Polymer Films. Langmuir 2011, 27, 14180-14187. [CrossRef] [PubMed]

24. He, G.S.; Yang, Z.J.; Pan, L.P.; Zhang, J.H.; Liu, S.J.; Yan, Q.L. Bioinspired interfacial reinforcement of polymer based energetic composites with a high loading of solid explosive crystals. J. Mater. Chem. A 2017, 5, 13499-13510. [CrossRef]

25. Gong, F.Y.; Zhang, J.H.; Ding, L.; Yang, Z.J.; Liu, X.B. Mussel-inspired coating of energetic crystals: A compact core-shell structure with highly enhanced thermal stability. Chem. Eng. J. 2017, 309, 140-150. [CrossRef] 
26. Lin, C.M.; Gong, F.Y.; Yang, Z.J.; Pan, L.P.; Liu, S.J.; Li, J.; Guo, S.Y. Bio-inspired fabrication of core@shell structured TATB/polydopamine microparticles via in situ polymerization with tunable mechanical properties. Polym. Test. 2018, 68, 126-134. [CrossRef]

27. Li, Y.B.; Yang, Z.J.; Zhang, J.H.; Pan, L.P.; Ding, L.; Tian, X.; Zheng, X.; Gong, F.Y. Fabrication and characterization of HMX@TPEE energetic microspheres with reduced sensitivity and superior toughness properties. Compos. Sci. Technol. 2017, 142, 253-263. [CrossRef]

28. Li, M.; Huang, M.; Kang, B.; Wen, M.P.; Li, H.Z.; Xu, R. Quality evaluation of RDX crystalline particles by confined quasi-static compression method. Propellants Explos. Pyrotech. 2007, 32, 401-405.

29. Young, R.A. The Rietveld Method; Oxford University Press: New York, NY, USA, 2002.

30. National Military Standard of China. Experimental Methods of Sensitivity and Safety; GJB/772A-97; National Defense Science Technology and Industry Comission Press: Beijing, China, 1997. (In Chinese)

31. Zangmeister, R.A.; Morris, T.A.; Tarlov, M.J. Characterization of Polydopamine Thin Films Deposited at Short Times by Autoxidation of Dopamine. Langmuir 2013, 29, 8619-8628. [CrossRef]

32. Lin, C.M.; Gong, F.Y.; Yang, Z.J.; Zhao, X.; Li, Y.B.; Zeng, C.C.; Li, J.; Guo, S.Y. Core-Shell Structured HMX@Polydopamine Energetic Microspheres: Synergistically Enhanced Mechanical, Thermal, and Safety Performances. Polymers 2019, 11, 568. [CrossRef]

33. Gong, F.Y.; Yang, Z.J.; Qjan, W.; Liu, Y.; Zhang, J.H.; Ding, L.; Lin, C.M.; Zeng, C.C.; Yan, Q.L. Kinetics for Inhibited Polymorphic Transition of HMX Crystal after Strong Surface Confinement. J. Phys. Chem. C 2019, 123, 11011-11019. [CrossRef]

34. Dartyge, B.F. Influence of crystal defects on sensitivity of explosives. In Proceedings of the 10th International Detonation Symposium, Boston, MA, USA, 12-16 June 1993; p. 816.

(C) 2020 by the authors. Licensee MDPI, Basel, Switzerland. This article is an open access article distributed under the terms and conditions of the Creative Commons Attribution (CC BY) license (http://creativecommons.org/licenses/by/4.0/). 